\title{
Lack of Evidence for Time or Dose Relationship between Antenatal Magnesium Sulfate and Intestinal Injury in Extremely Preterm Neonates
}

\author{
Michel Mikhael ${ }^{a, b}$ Cheryl Bronson ${ }^{a}$ Lishi Zhang ${ }^{c}$ Mark Curran $^{d}$ \\ Helen Rodriguez ${ }^{d} \quad$ Kushal Y. Bhakta ${ }^{a, b}$ \\ ${ }^{a}$ Division of Neonatology, Pomona Valley Hospital Medical Center, Pomona, CA, USA; ${ }^{\text {b }}$ Neonatal-Perinatal Medicine \\ Division, Children's Hospital of Orange County, Orange, CA, USA; ' Institute for Clinical and Translational Science, \\ University of California, Irvine, CA, USA; ${ }^{d}$ Division of Maternal Fetal Medicine, Pomona Valley Hospital Medical \\ Center, Pomona, CA, USA
}

\section{Keywords}

Magnesium sulfate $\cdot$ Necrotizing enterocolitis ·

Spontaneous intestinal perforation

\begin{abstract}
Background: Recent studies reported conflicting results on the relationship between antenatal magnesium sulfate $\left(\mathrm{MgSO}_{4}\right)$ exposure and neonatal intestinal injury. Most studies have not assessed $\mathrm{MgSO}_{4}$ exposure quantitatively and none reported the exposure timing. Objectives: The aim of this work was to assess whether there is a temporal or dosedependent relationship between antenatal $\mathrm{MgSO}_{4}$ exposure and intestinal injury in extremely preterm neonates. Methods: A retrospective study was made of inborn neonates with gestational age $\leq 28$ weeks and/or birth weights $\leq 1,000$ g. Primary outcomes included necrotizing enterocolitis (NEC), spontaneous intestinal perforation (SIP), and/or death prior to discharge or in the first 2 weeks of life. Outcome comparisons were made based on the timing of
\end{abstract}

$\mathrm{MgSO}_{4}$ exposure, within 7 days (Mg7D) or within 3 days (Mg3D) of birth. Total cumulative doses for the Mg3D group were also computed. Results: A total of 302 neonates were included, 210 in the Mg7D group, out of whom 179 (85.2\%) constituted the Mg3D group. There were no differences noted when comparing $\mathrm{MgSO}_{4}$ exposure timing and the likelihood of NEC, SIP, and/or death. This remained the same for subgroup analysis of neonates $<26$ weeks' gestation. Each 10-g increase in $\mathrm{MgSO}_{4}$ cumulative dose correlated with a decrease in SIP/NEC/death by $18.9 \%$ prior to discharge and by $21.9 \%$ in the first 2 weeks of life. Small for gestational age (SGA) was a potential effect modifier by a likelihood ratio test with $p=0.07$. Conclusions: Antenatal $\mathrm{MgSO}_{4}$ exposure in extremely preterm neonates was not associated with an increased risk of intestinal injury or death, and might have reduced these complications in a dose-dependent manner in our study. This protective effect was more noticeable in SGA neonates.

(c) 2019 S. Karger AG, Basel

\section{KARGER}

(c) 2019 S. Karger AG, Basel

E-Mail karger@karger.com

www.karger.com/neo
Michel Mikhael, MD

Neonatal-Perinatal Medicine Division, Children's Hospital of Orange County

1201 W. La Veta Avenue

Orange, CA 92868 (USA)

E-Mail mmikhael@choc.org 


\section{Introduction}

Magnesium sulfate $\left(\mathrm{MgSO}_{4}\right)$ has been used in obstetric practice for tocolysis, management of preeclampsia, and for fetal neuroprotection in women with threatened preterm delivery [1]. The latter practice has been widely adopted after the American College of Obstetricians and Gynecologists (ACOG) released a Committee Opinion in 2010 encouraging $\mathrm{MgSO}_{4}$ administration before anticipated early preterm birth to reduce the risk of cerebral palsy [2]. The recommendation was based on the results of three clinical trials that included 4,184 participants [3-5] and a Cochrane systematic review by Doyle et al. [6] in 2009. The systematic review concluded that the number of women needed to be treated to benefit 1 neonate by avoiding cerebral palsy is 63, without an increase in neonatal mortality or morbidities [6]. The ACOG, however, stated that comparison between the three major randomized clinical trials [3-5] was made difficult by differences in inclusion criteria, populations studied, $\mathrm{MgSO}_{4}$ regimens, and outcomes studied between the trials [2].

Subsequently, a number of groups have investigated the relationship between antenatal $\mathrm{MgSO}_{4}$ exposure and intestinal injury in premature neonates. In 2014, Rattray et al. [7] reported their experience with implementation of a protocol using $\mathrm{MgSO}_{4}$ for neuroprotection. They found a higher incidence of death or spontaneous intestinal perforation (SIP) during protocol implementation as compared to prior to implementation and after discontinuation ( 30.4 vs. $20.5 \%, p=0.28$ ), with a stronger correlation for higher $\mathrm{MgSO}_{4}$ dose and smaller neonates [7]. In a secondary analysis of the NICHD Maternal Fetal Medicine Units Network study [5], the authors reported there was a significantly elevated risk for death or severe necrotizing enterocolitis (NEC) in neonates with gestational age $(\mathrm{GA})<26$ weeks who were randomized to receive antenatal $\mathrm{MgSO}_{4}$, odds ratio $(\mathrm{OR})=1.9(95 \%$ CI $1.12-3.22, p=0.017$ [ [8]. In these two studies $\mathrm{MgSO}_{4}$ for neuroprotection regimens were in accordance with the NICHD Maternal Fetal Medicine Units Network study [5]. However, two larger reports by the Pediatrix Medical Group and the Canadian Neonatal Network showed no increase in neonatal adverse outcomes in relationship to any antenatal $\mathrm{MgSO}_{4}$ exposure $[9,10]$.

While $\mathrm{MgSO}_{4}$ infusion protocols vary among institutions in dose, duration, and criteria for reloading, most studies have assessed $\mathrm{MgSO}_{4}$ exposure in a dichotomous fashion only: exposed or not [7-10]. Moreover, no previous study has reported on the timing of antenatal
$\mathrm{MgSO}_{4}$ exposure in relation to birth or the onset of intestinal injury. Hence, it is difficult to arrive at a definitive conclusion regarding the association between antenatal $\mathrm{MgSO}_{4}$ exposure and neonatal intestinal injury. We aimed to explore these variables to determine if there is a temporal or dose-dependent relationship between antenatal $\mathrm{MgSO}_{4}$ exposure and neonatal intestinal injury in extremely preterm neonates.

\section{Methods}

\section{Study Population}

This was a retrospective study of neonates who were admitted to Pomona Valley Hospital Medical Center neonatal intensive care unit (NICU), Pomona, CA, USA, from January 2010 through December 2016. This is a tertiary level NICU with an annual average of 8,000 deliveries and 800 NICU admissions. Subjects included inborn neonates with birth weight $\leq 1,000 \mathrm{~g}$ and/or GA $\leq 28$ weeks with no congenital gastrointestinal anomalies. The study was approved by the hospital institutional review board.

\section{$\mathrm{MgSO}_{4}$ Protocol}

In October 2012, $\mathrm{MgSO}_{4}$ for the neuroprotection protocol was implemented in our center. Women with threatened preterm delivery at 24-32 weeks' gestation receive an $\mathrm{MgSO}_{4}$ loading dose of $4 \mathrm{~g}$ over $30 \mathrm{~min}$, followed by an infusion of $2 \mathrm{~g} / \mathrm{h}$ for $12 \mathrm{~h}$ or until delivery. If delivery does not occur by $12 \mathrm{~h}$, the infusion is stopped and resumed if delivery is thought to be imminent. Also, a repeat loading dose is administered if $>6 \mathrm{~h}$ has passed after discontinuation of the infusion.

\section{$\mathrm{MgSO}_{4}$ Exposure}

The following exposure groups were chosen to describe the different times of antenatal $\mathrm{MgSO}_{4}$ exposure in relation to birth: neonates who received $\mathrm{MgSO}_{4}$ within 7 days $(\mathrm{Mg} 7 \mathrm{D})$ or within 3 days (Mg3D) prior to birth. Among patients who delivered within 3 days, the number of $\mathrm{MgSO}_{4}$ loading doses and cumulative doses were also compared to delineate any potential dose-dependent effect.

\section{Outcomes of Interest}

Primary outcomes included SIP (diagnosed intraoperatively or radiologically), NEC (Bell's staging criteria II or greater) [11], and death, either individually or combined. We analyzed these outcomes prior to hospital discharge or within 2 weeks of life. We also examined the likelihood of NEC, SIP, or death before and after $\mathrm{MgSO}_{4}$ for neuroprotection protocol implementation.

\section{Statistical Methods}

For baseline characteristics, $t$ test was used for continuous variables and $\chi^{2}$ test for categorical variables. For the univariate analysis, $\chi^{2}$ test was used to compare both individual and combined outcomes between exposure groups. The univariate association was examined for the entire cohort as well as the subcohort with $\mathrm{GA}<26$ weeks. The $\mathrm{MgSO}_{4}$ exposure effect was further examined with a multivariate logistic regression analysis after adjusting for significantly different baseline characteristics. Logistic regression 
Fig. 1. Study population.

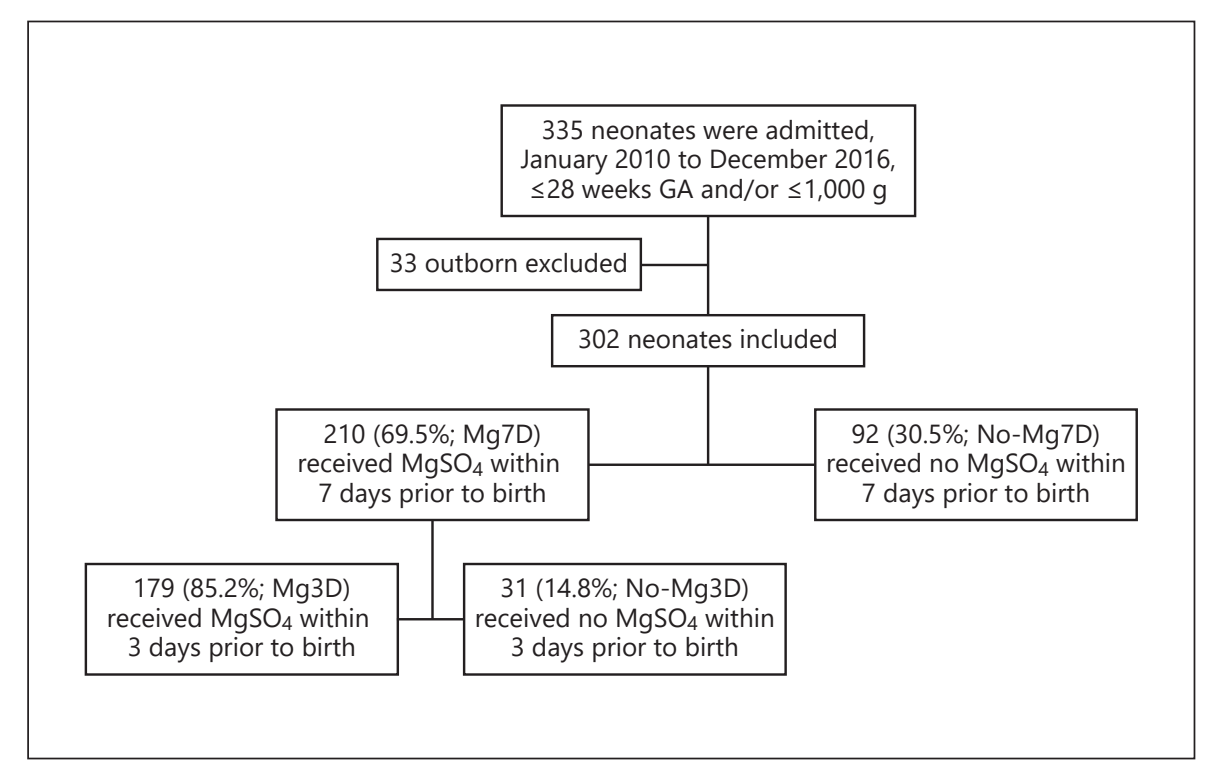

Table 1. Maternal, perinatal, and neonatal characteristics of the subjects

\begin{tabular}{|c|c|c|c|}
\hline & $\begin{array}{l}\mathrm{Mg} 7 \mathrm{D} \\
(n=210)\end{array}$ & $\begin{array}{l}\text { No-Mg7D } \\
(n=92)\end{array}$ & $p$ value \\
\hline \multicolumn{4}{|l|}{ Maternal characteristics } \\
\hline Age, years & $28.1 \pm 6.4$ & $27.5 \pm 6.5$ & 0.43 \\
\hline Body mass index & $31.3 \pm 6.6$ & $29.9 \pm 7.8$ & 0.14 \\
\hline Race & & & 0.58 \\
\hline White & $40(19)$ & $14(15.2)$ & \\
\hline Black & $28(13.3)$ & $16(17.4)$ & \\
\hline Hispanic & $112(53.3)$ & $52(56.5)$ & \\
\hline Other & $30(14.2)$ & $10(10.9)$ & \\
\hline Illicit drug abuse & $21(10)$ & $10(10.9)$ & 0.82 \\
\hline Maternal hypertension & $62(29.5)$ & $13(14.1)$ & 0.004 \\
\hline \multicolumn{4}{|l|}{ Perinatal characteristics } \\
\hline Prolonged rupture of membranes & $54(25.7)$ & $24(26.1)$ & 0.95 \\
\hline Cesarean delivery & $146(69.5)$ & $67(72.8)$ & 0.56 \\
\hline Antenatal steroids & $200(95.2)$ & $59(64.1)$ & $<0.0001$ \\
\hline Antenatal indomethacin & $43(20.5)$ & $8(8.7)$ & 0.012 \\
\hline Multiple gestations & $31(14.8)$ & $20(21.7)$ & 0.14 \\
\hline \multicolumn{4}{|l|}{ Neonatal characteristics } \\
\hline Gestational age, weeks & $26.2 \pm 1.9$ & $26.1 \pm 2.1$ & 0.6 \\
\hline Birth weight, $g$ & $804 \pm 212$ & $812 \pm 231$ & 0.8 \\
\hline Male sex & $101(48.1)$ & $48(52.2)$ & 0.5 \\
\hline 5-min Apgar score & $7(6-8)$ & $7(5-8)$ & 0.078 \\
\hline SGA & $36(17.1)$ & $14(15.2)$ & 0.68 \\
\hline Postnatal steroids & $79(37.6)$ & $37(40.2)$ & 0.67 \\
\hline Postnatal NSAID treatment for PDA & $73(34.76)$ & $40(43.48)$ & 0.15 \\
\hline First serum magnesium level within 72 h, mg/dL & $2.7 \pm 0.9$ & $2.1 \pm 0.4$ & $<0.0001$ \\
\hline Intraventricular hemorrhage grade III or higher & $19(9)$ & $11(12)$ & 0.4 \\
\hline Late-onset sepsis & $42(20)$ & $27(29.3)$ & 0.075 \\
\hline
\end{tabular}

Data are presented as the mean $\pm \mathrm{SD}, n(\%)$, or median (IQR). SGA, small for gestational age; NSAID, nonsteroidal anti-inflammatory drugs; PDA, patent ductus arteriosus. 
Table 2. Comparison of outcomes among different exposure groups for all neonates and neonates with GA $<26$ weeks

\begin{tabular}{|c|c|c|c|c|c|c|c|c|c|}
\hline & Mg7D & No-Mg7D & $\begin{array}{l}p \\
\text { value }\end{array}$ & $\mathrm{Mg} 3 \mathrm{D}$ & $\begin{array}{l}\text { No-Mg7D + } \\
\text { No-Mg3D }\end{array}$ & $\begin{array}{l}p \\
\text { value }\end{array}$ & $\operatorname{Mg} 3 \mathrm{D}$ & No-Mg3D & $\begin{array}{l}p \\
\text { value }\end{array}$ \\
\hline All neonates & $(n=210)$ & $(n=92)$ & & $(n=179)$ & $(n=123)$ & & $(n=179)$ & $(n=31)$ & \\
\hline SIP & $20(9.5)$ & $8(8.7)$ & 0.82 & $18(10.1)$ & $10(8.1)$ & 0.57 & $18(10.1)$ & $2(6.4)$ & 0.53 \\
\hline Early SIP & $19(9.1)$ & $7(7.6)$ & 0.68 & $17(9.5)$ & $9(7.3)$ & 0.54 & $17(9.5)$ & $2(6.5)$ & 0.75 \\
\hline NEC & $15(7.1)$ & $7(7.6)$ & 0.89 & $13(7.3)$ & $9(7.3)$ & 0.98 & $13(7.3)$ & $2(6.5)$ & 0.87 \\
\hline Early death & $15(7.1)$ & $10(10.9)$ & 0.28 & $13(7.3)$ & $12(9.8)$ & 0.44 & $13(7.3)$ & $2(6.5)$ & 0.87 \\
\hline SIP/NEC/death & $52(24.8)$ & $24(26.1)$ & 0.81 & $43(24)$ & $33(26.8)$ & 0.58 & $43(24)$ & $9(29)$ & 0.55 \\
\hline Early SIP/NEC/death & $34(16.2)$ & $16(17.4)$ & 0.79 & $29(16.2)$ & $21(17.1)$ & 0.8412 & $29(16.2)$ & $5(16.1)$ & 0.99 \\
\hline Neonates with $G A<26$ weeks & $(n=90)$ & $(n=45)$ & & $(n=75)$ & $(n=60)$ & & $(n=75)$ & $(n=15)$ & \\
\hline SIP & $14(15.6)$ & $6(13.3)$ & 0.73 & $13(17.3)$ & $7(11.7)$ & 0.36 & $13(17.3)$ & $1(6.7)$ & 0.3 \\
\hline Early death & $12(13.3)$ & $9(20)$ & 0.31 & $10(13.3)$ & $11(18.3)$ & 0.43 & $10(13.3)$ & $2(13.3)$ & 1.0 \\
\hline SIP/NEC/death & $34(37.8)$ & $20(44.4)$ & 0.46 & $27(36)$ & $27(45)$ & 0.29 & $27(36)$ & $7(46.7)$ & 0.44 \\
\hline Early SIP/NEC/death & $26(28.9)$ & $13(28.9)$ & 1.0 & $22(29.3)$ & $17(28.3)$ & 0.9 & $22(29.3)$ & $4(26.7)$ & 0.83 \\
\hline
\end{tabular}

Data are presented as $n(\%)$. SIP, spontaneous intestinal perforation; NEC, necrotizing enterocolitis.

modeling was used to examine the effect of $\mathrm{MgSO}_{4}$ loads and cumulative doses on the outcomes among neonates who were exposed to antenatal $\mathrm{MgSO}_{4}$ within 3 days prior to birth. Additionally, effect modification for all baseline characteristics was assessed with likelihood ratio tests.

\section{Results}

\section{Baseline Characteristics}

A total of 302 neonates met the inclusion criteria. From these, $210(69.5 \%)$ were exposed to antenatal $\mathrm{MgSO}_{4}$ within 7 days prior to birth, out of whom 179 $(85.2 \%)$ were exposed within 3 days prior to birth (Fig. 1).

The subjects' maternal, perinatal, and neonatal characteristics (Table 1) were compared between the Mg7D group and the group of neonates not exposed to antenatal $\mathrm{MgSO}_{4}$ within 7 days prior to birth (No-Mg7D). The Mg7D group had a significantly higher likelihood of maternal hypertension, exposure to antenatal steroids, and antenatal indomethacin. Also, a higher neonatal first serum magnesium level (when measured within $72 \mathrm{~h}$ of life) was detected in the Mg7D group. There were no other significant differences in perinatal or neonatal characteristics between the Mg7D and NoMg7D groups that might contribute to intestinal injury (Table 1).

\section{$\mathrm{MgSO}_{4}$ Time of Exposure and Outcomes}

There were no differences noted between different $\mathrm{MgSO}_{4}$ exposure timings and the likelihood of SIP, NEC, or death prior to hospital discharge or in the first 2 weeks of life (early). Combined outcomes also did not show significant differences. This was true for the entire cohort and in a subgroup analysis of neonates $<26$ weeks' gestation (Table 2).

\section{Multivariate Analysis on Combined Outcomes}

Maternal hypertension, antenatal steroids, and antenatal indomethacin were adjusted in the multivariate logistic regression (Table 3 ) on the combined outcome of SIP/NEC/death due to the differences observed in baseline characteristics comparisons (Table 1). After controlling for potential confounding variables there were no significant differences in the odds of SIP/NEC/death between the $\mathrm{Mg} 7 \mathrm{D}$ and No-Mg7D groups prior to discharge, $\mathrm{OR}=0.69$ (95\% CI 0.35-1.38, $p=0.29$ ). This remained similar for early SIP/NEC/death as well with $\mathrm{OR}=1.7$ (95\% CI 0.73-3.75, $p=0.22$; Table 3$)$. 
Table 3. Results of multivariate logistic regression

\begin{tabular}{|c|c|c|c|c|c|}
\hline Variable & Category & Parameter & SE & OR (95\% CI) & $p$ value \\
\hline \multicolumn{6}{|c|}{ Effect on SIP/NEC/death prior to discharge } \\
\hline Mg7D & $\begin{array}{l}\text { Yes } \\
\text { No }\end{array}$ & $\begin{array}{l}-0.3664 \\
\quad \text { (reference) }\end{array}$ & 0.3494 & $0.69(0.35-1.38)$ & 0.29 \\
\hline Maternal hypertension & & -0.8426 & 0.3879 & $0.43(0.2-0.92)$ & 0.03 \\
\hline Antenatal steroids & & -1.2559 & 0.4061 & $0.28(0.13-0.63)$ & 0.002 \\
\hline Antenatal indomethacin & & 0.8936 & 0.3441 & $2.44(1.24-4.8)$ & 0.009 \\
\hline \multicolumn{6}{|c|}{ Effect on SIP/NEC/death in the first 2 weeks of life } \\
\hline Mg7D & $\begin{array}{l}\text { Yes } \\
\text { No }\end{array}$ & $\begin{array}{l}0.5067 \\
\text { (reference) }\end{array}$ & 0.4159 & $1.7(0.73-3.75)$ & 0.22 \\
\hline Maternal hypertension & & -0.7547 & 0.4532 & $0.47(0.19-1.14)$ & 0.096 \\
\hline Antenatal steroids & & -1.4523 & 0.4500 & $0.23(0.10-0.57)$ & 0.0012 \\
\hline Antenatal indomethacin & & 0.5568 & 0.4010 & $1.75(0.80-3.83)$ & 0.16 \\
\hline
\end{tabular}

Fig. 2. Comparison of early SIP/NEC/ death ORs for Mg7D versus No-Mg7D among all, SGA, and non-SGA neonates.

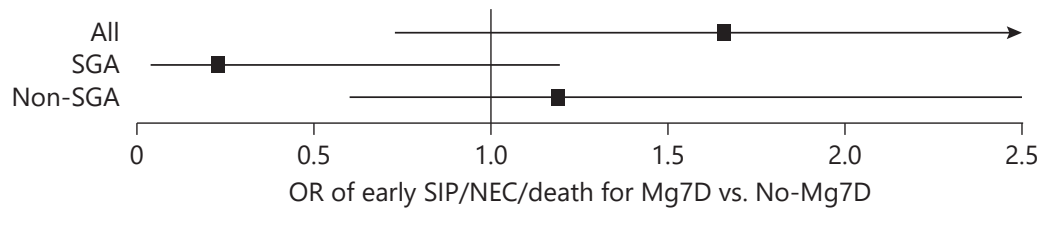

Before and after $\mathrm{MgSO}_{4}$ for Neuroprotection Protocol Implementation Analysis

The study included 112 neonates born before and 190 born after the protocol implementations. Subjects' maternal, perinatal, and neonatal characteristics showed no significant differences except the higher rate of exposure to antenatal $\mathrm{MgSO}_{4}$ within 7 days of birth in the postprotocol group (pre. vs. post., 58.9 vs. $75.8 \%, p=0.003$ ). There was no difference in the rate of exposure to antenatal $\mathrm{MgSO}_{4}$ within 3 days of birth (pre. vs. post., 53.5 vs. $62.6 \%, p=0.15$ ).

The likelihoods of intestinal injury and death were similar in the pre- versus post-protocol groups: SIP (10.7 vs. $8.4 \%, p=0.6)$, NEC ( 8.9 vs. $6.3 \%, p=0.5)$, or death $(8.9$ vs. $13.7 \%, p=0.3$ ). Among the 135 neonates with GA $<26$ weeks, 49 were born before and 86 after protocol implementation. The likelihood of adverse outcomes was not significantly different in the pre- versus post-protocol groups: SIP (22.4 vs. $10.4 \%, p=0.1$ ), NEC (12.2 vs. 9.3\%, $p=0.8$ ), or death (16.3 vs. $23.2 \%, p=0.4$ ).

$\mathrm{MgSO}_{4}$ Is Not Associated with Intestinal Injury

\section{$\mathrm{MgSO}_{4}$ Dose-Dependent Effect}

We examined the effect of $\mathrm{MgSO}_{4}$ cumulative dose on the combined outcomes of SIP/NEC/death among the 179 neonates exposed to antenatal $\mathrm{MgSO}_{4}$ within 3 days prior to birth $(\mathrm{Mg} 3 \mathrm{D})$. Based on logistic regression modeling, each 10-g increase in $\mathrm{MgSO}_{4}$ cumulative dose correlated with an $18.9 \%$ decrease in SIP/NEC/death prior to discharge (95\% CI $2.2-32.8 \%, p=0.028$ ) and a $21.9 \%$ decrease in early SIP/NEC/death (95\% CI 1.4-38.1\%, $p=$ 0.037).

However, there was no significant association between the number of $\mathrm{MgSO}_{4}$ loading doses and SIP/NEC/death prior to discharge, $\mathrm{OR}=1.07$ (95\% CI 0.53-2.1, $p=0.86$ ), or early SIP/NEC/death, OR = 1.08 (95\% CI 0.48-2.4, $p=$ $0.86)$.

\section{Assessment on Effect Modification}

Small for GA (SGA) status, defined as neonates with a birth weight below the 10th percentile for GA using Fenton and Kim [12] growth curves, was found to be 
correlated with a reduction in SIP/NEC/ death in the $\mathrm{Mg} 7 \mathrm{D}$ group by a likelihood ratio test with $p=0.07$. The OR of early SIP/NEC/death for Mg7D versus No$\mathrm{Mg} 7 \mathrm{D}$ in SGA neonates was 0.23 (95\% CI 0.04-1.19, $p=0.079$ ). There was no difference demonstrated in the non-SGA neonates with OR $=1.19$ (95\% CI 0.58$2.47, p=0.63$; Fig. 2 ). The significance level of treatment effect in the SGA group was around the boundary $(p<0.1)$.

\section{Discussion}

Recent studies $[7,8]$ have raised concerns regarding the association between antenatal $\mathrm{MgSO}_{4}$ exposure and intestinal injury or death in preterm neonates. In our cohort of 302 neonates with a birth weight $\leq 1,000 \mathrm{~g}$ and/ or GA $\leq 28$ weeks, we found no relationship between antenatal $\mathrm{MgSO}_{4}$ exposure and the individual or combined outcomes of SIP, NEC, or death. This remained true for the subset of neonates $<26$ weeks GA. Our results complement data from the studies by the Pediatrix Medical Group [9] and the Canadian Neonatal Network [10]. Similarly, the original randomized controlled trials investigating antenatal $\mathrm{MgSO}_{4}$ for neuroprotection [3-5] showed no increase in neonatal and pediatric adverse outcomes.

This is the first study to examine the temporal relationship between antenatal $\mathrm{MgSO}_{4}$ exposure and adverse neonatal outcomes. $\mathrm{MgSO}_{4}$ decreases intestinal peristalsis [13], can possibly alter mesenteric blood flow $[14,15]$, and is associated with feeding intolerance in neonates [16]. It also crosses the placenta freely with a linear relationship between maternal and neonatal levels [17-19]. However, neonatal intestinal injury and death can be caused by many contributing factors. Therefore, it was compelling for us to investigate if there is a temporal relationship between antenatal $\mathrm{MgSO}_{4}$ exposure and the onset of intestinal injury or death. We found no association between different timing of antenatal $\mathrm{MgSO}_{4}$ exposure (Mg7D and Mg3D) and the likelihood of an adverse outcome either prior to hospital discharge or in the first 2 weeks of life.

In contrast to the study by Rattray et al. [7], we observed no increase in the likelihood of SIP or death after the implementation of $\mathrm{MgSO}_{4}$ for the neuroprotection protocol at our hospital. Moreover, we found that an increased $\mathrm{MgSO}_{4}$ cumulative dose correlated with a reduction in SIP/NEC/death both early and prior to discharge. Our study included a larger population with a longer post-protocol duration and a similar rate of exposure to antenatal $\mathrm{MgSO}_{4}$ post-protocol.

It is noteworthy that the post-protocol group had a higher rate of exposure to antenatal $\mathrm{MgSO}_{4}$ within 7 days of birth (pre. vs. post. 58.95 vs. $75.8 \%, p=0.003$ ) but not within 3 days of birth (pre. vs. post. 53.5 vs. $62.6 \%, p=0.15$ ), probably as the indication for 7 days of exposure was primarily for fetal neuroprotection.

Downey et al. [9] reported fewer SIP/NEC/deaths in neonates exposed to any antenatal $\mathrm{MgSO}_{4}$ with $\mathrm{OR}=$ 0.84 (95\% CI 0.77-0.9), which our results did not reproduce. However, we did find that each 10 -g increase in $\mathrm{MgSO}_{4}$ cumulative dose correlated with an $18.9 \%$ decrease in SIP/NEC/death prior to discharge (95\% CI $2.2-32.8 \%, p=0.028)$ and a $21.9 \%$ decrease in early SIP/ NEC/death (95\% CI $1.4-38.1 \%, p=0.037)$. Their results were primarily driven by a reduction in death among neonates exposed to $\mathrm{MgSO}_{4}, \mathrm{OR}=0.76$ (95\% CI 0.70 0.83) [9]. This differs from the Cochrane review by Doyle et al. [6], which reported no difference in neonatal death during primary hospitalization, OR $=1.04$ (95\% CI 0.84-1.29). However, the Downey et al. [9] study had a significantly larger population $(28,035$ vs. 5,980$)$ with an average lower birth weight, including only neonates $<1,000 \mathrm{~g}$.

SGA represents a high-risk population for neonatal mortality and morbidities, including intestinal injury [20-22]. A possible protective effect of antenatal $\mathrm{MgSO}_{4}$ in SGA neonates (Fig. 2) was an unexpected finding which was not previously reported in the literature. Marrs et al. [23] recently performed a secondary analysis of the NICHD Maternal Fetal Medicine Units Network randomized controlled trial of $\mathrm{MgSO}_{4}$ for the prevention of cerebral palsy [5]. In their report, SGA neonates had similar rates of neonatal morbidities and mortality in comparison to non-SGA neonates. It is noteworthy that $54 \%$ of SGA neonates were randomized to receive antenatal $\mathrm{MgSO}_{4}$ in this study [23]. We speculate that $\mathrm{MgSO}_{4}$ exposure could have contributed to better neonatal outcomes in comparison to earlier studies [20-22].

This study's limitations include its retrospective nature and that it was performed at a single center. The chosen exposure timing of within 3 or 7 days of birth were exploratory, as previous studies did not report on the temporal relationship of $\mathrm{MgSO}_{4}$ exposure and neonatal outcomes. Future studies might focus on narrower exposure timing, such as less than $24 \mathrm{~h}$. Another limitation is the relatively small number of SGA neonates included in the analysis. 
However, our report has unique strengths, including analyzing the cumulative dose and exposure timing of $\mathrm{MgSO}_{4}$ in relation to birth, reporting on a contemporary cohort of neonates with no other practice changes that might have affected neonatal outcomes and complete outcome data without missing endpoints.

The present study supports the likelihood that antenatal $\mathrm{MgSO}_{4}$ exposure in preterm neonates does not increase the risk of intestinal injury or death and may in fact reduce these complications at higher doses. This protective effect was more noticeable in SGA neonates. Future studies might clarify the treatment effects in this subgroup.

\section{Statement of Ethics}

The study was approved by the institutional review board of Pomona Valley Hospital Medical Center, Pomona, CA, USA. Informed consents were not required since it is a retrospective study.

\section{Disclosure Statement}

The authors have no conflicts of interest to declare.

\section{Funding Sources}

This work was partially supported by grant UL1 TR001414 from the National Center for Advancing Translational Sciences, National Institutes of Health (NIH), through the Biostatistics, Epidemiology and Research Design Unit. The content is solely the responsibility of the authors and does not necessarily represent the official views of the NIH.

\section{Author Contributions}

M.M. contributed to the conceptualization and design of the study, the collection, analysis, and interpretation of the data. $\mathrm{He}$ drafted the initial report and approved the final manuscript as submitted. C.B. contributed to the design of the study and the data collection. She reviewed all drafts of the report and approved the final manuscript as submitted. L.Z. contributed to the analysis and interpretation of the data. She contributed to all drafts of the report and approved the final manuscript as submitted. M.C. and H.R. contributed to the interpretation of the data. They contributed to all drafts of the report and approved the final manuscript as submitted. K.Y.B contributed to the conceptualization and design of the study, and the collection and interpretation of the data. $\mathrm{He}$ contributed to all drafts of the report and approved the final manuscript as submitted.

\section{References}

1 Pryde PG, Mittendorf R. Contemporary usage of obstetric magnesium sulfate: indication, contraindication, and relevance of dose. Obstet Gynecol. 2009 Sep;114(3):669-73.

2 American College of Obstetricians and Gynecologists Committee on Obstetric Practice; Society for Maternal-Fetal Medicine. Committee Opinion No. 455: magnesium sulfate before anticipated preterm birth for neuroprotection. Obstet Gynecol. 2010 Mar;115(3): 669-71.

3 Crowther CA, Hiller JE, Doyle LW, Haslam RR; Australasian Collaborative Trial of Magnesium Sulphate (ACTOMg SO4) Collaborative Group. Effect of magnesium sulfate given for neuroprotection before preterm birth: a randomized controlled trial. JAMA. 2003 Nov;290(20):2669-76.

4 Marret S, Marpeau L, Zupan-Simunek V, Eurin $\mathrm{D}$, Lévêque $\mathrm{C}$, Hellot $\mathrm{MF}$, et al.; PREMAG trial group. Magnesium sulphate given before very-preterm birth to protect infant brain: the randomised controlled PREMAG trial. BJOG. 2007 Mar;114(3): $310-8$.

$\mathrm{MgSO}_{4}$ Is Not Associated with Intestinal Injury
5 Rouse DJ, Hirtz DG, Thom E, Varner MW Spong CY, Mercer BM, et al.; Eunice Kennedy Shriver NICHD Maternal-Fetal Medicine Units Network. A randomized, controlled trial of magnesium sulfate for the prevention of cerebral palsy. N Engl J Med. 2008 Aug; 359(9):895-905.

6 Doyle LW, Crowther CA, Middleton P, Marret S, Rouse D. Magnesium sulphate for women at risk of preterm birth for neuroprotection of the fetus. Cochrane Database Syst Rev. 2009 Jan;1:CD004661.

7 Rattray BN, Kraus DM, Drinker LR, Goldberg RN, Tanaka DT, Cotten CM. Antenatal magnesium sulfate and spontaneous intestinal perforation in infants less than 25 weeks gestation. J Perinatol. 2014;34(11):819-22.

8 Kamyar M, Clark EA, Yoder BA, Varner MW, Manuck TA. Antenatal magnesium sulfate, necrotizing enterocolitis, and death among neonates $<28$ weeks gestation. AJP Rep. 2016 Mar;6(1):e148-54.

9 Downey LC, Cotten CM, Hornik CP, Laughon MM, Tolia VN, Clark RH, et al. Association of in utero magnesium exposure and spontaneous intestinal perforations in extremely low birth weight infants. J Perinatol. 2017;37(6): 641-4.
10 Shalabi M, Mohamed A, Lemyre B, Aziz K, Faucher D, Shah PS; Canadian Neonatal Network Investigators. Antenatal Exposure to Magnesium Sulfate and Spontaneous Intestinal Perforation and Necrotizing Enterocolitis in Extremely Preterm Neonates. Am J Perinatol. 2017 Oct;34(12):1227-33.

11 Bell MJ, Ternberg JL, Feigin RD, Keating JP, Marshall R, Barton L, et al. Neonatal necrotizing enterocolitis. Therapeutic decisions based upon clinical staging. Ann Surg. 1978 Jan; 187(1):1-7.

12 Fenton TR, Kim JH. A systematic review and meta-analysis to revise the Fenton growth chart for preterm infants. BMC Pediatr. 2013 Apr;13(1):59.

$13 \mathrm{Lu} J \mathrm{~F}$, Nightingale CH. Magnesium sulfate in eclampsia and pre-eclampsia: pharmacokinetic principles. Clin Pharmacokinet. 2000 Apr;38(4):305-14.

14 Havranek T, Ashmeade TL, Afanador M Carver JD. Effects of maternal magnesium sulfate administration on intestinal blood flow velocity in preterm neonates. Neonatology. 2011;100(1):44-9. 
15 Gursoy T, Imamoglu EY, Ovali F, Karatekin G. Effects of antenatal magnesium exposure on intestinal blood flow and outcome in preterm neonates. Am J Perinatol. 2015 Sep; 32(11):1064-9.

16 Belden MK, Gnadt S, Ebert A. Effects of Maternal Magnesium Sulfate Treatment on Neonatal Feeding Tolerance. J Pediatr Pharmacol Ther. 2017 Mar-Apr;22(2):112-7.

17 Hallak M, Berry SM, Madincea F, Romero R, Evans MI, Cotton DB. Fetal serum and amniotic fluid magnesium concentrations with maternal treatment. Obstet Gynecol. 1993 Feb;81(2):185-8.
18 Brookfield KF, Su F, Elkomy MH, Drover DR, Lyell DJ, Carvalho B. Pharmacokinetics and placental transfer of magnesium sulfate in pregnant women. Am J Obstet Gynecol. 2016; 214(6):737.e1-9.

19 García Alonso L, Pumarada Prieto M, González Colmenero E, Concheiro Guisán A, Suárez Albo M, Durán Fernández-Feijoo C, et al. Prenatal Therapy with Magnesium Sulfate and Its Correlation with Neonatal Serum Magnesium Concentration. Am J Perinatol. 2018 Jan;35(2):170-6.

20 McIntire DD, Bloom SL, Casey BM, Leveno $\mathrm{KJ}$. Birth weight in relation to morbidity and mortality among newborn infants. N Engl J Med. 1999 Apr;340(16):1234-8.
21 Garite TJ, Clark R, Thorp JA. Intrauterine growth restriction increases morbidity and mortality among premature neonates. Am J Obstet Gynecol. 2004 Aug;191(2):481-7.

22 Grobman WA, Lai Y, Rouse DJ, Spong CY, Varner MW, Mercer BM, et al. The association of cerebral palsy and death with smallfor-gestational-age birthweight in preterm neonates by individualized and populationbased percentiles. Am J Obstet Gynecol. 2013; 209(4):340.e1-5.

23 Marrs CC, Mendez-Figueroa H, Hammad IA, Chauhan SP. Differential Morbidity in Preterm Small versus Appropriate for Gestational Age: perhaps Unverifiable. Am J Perinatol. 2015 Nov;32(13):1251-6. 